\title{
Eficiência de Utilização da Energia Metabolizável para Mantença e Ganho de Peso e Exigências de Energia Metabolizável e de Nutrientes Digestíveis Totais de Bovinos Nelore, Não-Castrados
}

\section{Antonia Sherlânea Chaves Véras ${ }^{2}$, Sebastião de Campos Valadares Filho ${ }^{3}$, José Fernando Coelho da Silva ${ }^{4}$, Mário Fonseca Paulino ${ }^{3}$, Paulo Roberto Cecon ${ }^{5}$, Marcelo de Andrade Ferreira $^{2}$, Rilene Ferreira Diniz Valadares ${ }^{6}$, Eduardo Henrique Bevitori Kling de Moraes ${ }^{7}$}

RESUMO - Este trabalho foi conduzido para estimar as eficiências de utilização da energia metabolizável (EUEM) para mantença $(\mathrm{km})$ e ganho de peso (kf) e as exigências de energia metabolizável (EM) e nutrientes digestíveis totais (NDT) de bovinos Nelore, nãocastrados. Utilizaram-se 35 animais com idade e peso médios de 20 meses e $330 \mathrm{~kg}$. Após período de adaptação ( 40 dias), cinco animais foram abatidos para servir como referência; os demais foram distribuídos, em delineamento inteiramente casualizado, nos tratamentos $(12,5 ; 25,0 ; 37,5 ; 50,0 ; 62,5 ;$ e 75,0\% de concentrado) com base na matéria seca. A estimativa das kf foi efetuada a partir da regressão entre os teores de energia líquida para ganho, em função da EM da dieta, e também agrupando-se os dados relativos aos animais do presente trabalho e os referentes a 29 bovinos F1 Simental x Nelore. As kf também foram estimadas como o coeficiente da regressão linear entre a energia retida (ER) e o consumo de EM (CEM). A km estimada foi de 0,56. As kf estimadas utilizando-se a primeira metodologia foram de 0,$45 ; 0,35 ; 0,54 ; 0,50$; e 054 , respectivamente, para as concentrações de 2,$11 ; 2,35 ; 2,51 ; 2,59$; e 2,52 Mcal/ $\mathrm{kg}$ de MS. As kf estimadas por meio da segunda metodologia foram 0,$32 ; 0,33 ; 0,44 ; 0,49$; e 0,69 , respectivamente. Os requisitos de EM e NDT para mantença de um animal pesando $400 \mathrm{~kg}$ de PV foram de 11,94 Mcal/kgGPCVZ e 3,30 kg/kgGPCVZ, respectivamente; enquanto para ganho de peso, os requisitos para dietas com 2,4 Mcal/kg de MS, foram 9,84 Mcal/kg GPCVZ e 2,72 kg/GPCVZ. Para dietas com concentração de EM de 2,6 Mcal/kg de MS, os requerimentos de EM e NDT foram, respectivamente, $7,28 \mathrm{Mcal} / \mathrm{kg} \mathrm{GPCVZ}$ e 2,01 kg/GPCVZ.

Palavras-chave: concentrado, eficiência, energia metabolizável, nutrientes digestíveis totais

\section{Efficiency of Metabolizable Energy Utilization for Maintenance and Weight Gain and Requirements of Metabolizable Energy and Total Digestible Nutrients of Nellore Bulls}

ABSTRACT - This work was carried out to estimate the efficiency of metabolizable energy utilization (EMEU) for maintenance $(\mathrm{km})$ and weight gain $(\mathrm{kf})$ and the requirements of metabolizable energy (ME) and total digestible nutrients (TDN) of Nellore bulls. Thirtyfive animals, averaging $330 \mathrm{~kg}$ and 20 months old, were used. After the period of adaptation (40 days), five animals were slaughtered as reference. The remaining animals were allotted, in a completely randomized design, to the treatments (12.5, 25.0, 37.5, 50.0, 62.5, and $75.0 \%$ of concentrate), in dry matter basis. The kf estimate was effected from the regression among net energy contents for gain, in function of dietary ME, and also grouping data relative to the animals of the present work and the ones of the $29 \mathrm{~F} 1 \mathrm{Simental} \mathrm{x}$ Nellore bulls. The kf were also estimated as the coefficient of the linear regression between the retained energy (RE) and the ME intake (MEI). The estimated $\mathrm{km}$ was .56. The estimated $\mathrm{kf}$ using the first methodology were $.45, .35, .54, .50$, and .54 , respectively, for the concentrations of $2.11,2.35,2.51,2.59$, and $2.52 \mathrm{Mcal} / \mathrm{kg} \mathrm{DM}$. The $\mathrm{kf}$ estimated by means of the second methodology were .32 , $.33, .44, .49$, and .69 , respectively. The ME and TDN requirements to maintain a $400 \mathrm{~kg} \mathrm{LW}$ animal were $11.94 \mathrm{Mcal} / \mathrm{kg}$ EBWG and $3.30 \mathrm{~kg} / \mathrm{kg}$ EBWG, respectively, while for weight gain, the requirements for the diets with $2.4 \mathrm{Mcal} / \mathrm{kg} \mathrm{DM}$, were $9.84 \mathrm{Mcal} / \mathrm{kg}$ EBWG and $2.72 \mathrm{~kg} / \mathrm{EBWG}$. For the diets with ME concentration of $2.6 \mathrm{Mcal} / \mathrm{kg}$ DM, the ME and TDN requirements were $7.28 \mathrm{Mcal} / \mathrm{kg}$ EBWG and $2.01 \mathrm{~kg} / \mathrm{EBWG}$, respectively.

Key Words: concentrate, efficiency, metabolizable energy, total digestible nutrients

\footnotetext{
${ }^{1}$ Parte da tese de Doutorado do primeiro autor, parcialmente financiada pela FINEP.

2 Professor do DZ/UFRPE. E.mail: sherlanea@uol.com.br

3 Professor do DZO/UFV.

${ }_{5}^{4}$ Professor do LZNA-CCTA/UENF.

5 Professor do DPI/UFV.

6 Professor do DMV/UFV.

7 Aluno de graduação em Zootecnia/UFV.
} 


\section{Introdução}

A partir do conhecimento dos requerimentos líquidos de energia e dos fatores de eficiência de utilização da energia do alimento para mantença e ganho, são obtidas as exigências dietéticas. Portanto, o conhecimento da eficiência de utilização da energia metabolizável (EUEM) da dieta é necessário para a determinação das exigências de energia metabolizável.

As estimativas das EUEM para mantença $(\mathrm{km}) \mathrm{e}$ ganho (kf) são obtidas a partir de equações de terceiro grau entre a energia líquida de mantença (ELm) e a energia metabolizável (EM) da dieta e entre a energia líquida para ganho (ELg) e a EM da dieta, respectivamente, com sua determinação obtida pelas relações entre as ELm ou ELg estimadas e a EM da dieta (GARRETT, 1980a,b). A kf também pode ser estimada como o coeficiente da regressão linear entre a energia retida (ER) e o consumo de energia metabolizável (CEM), segundo o NATIONAL RESEARCH COUNCIL - NRC (1996) e FERRELL e JENKINS (1998a,b).

Para COELHO DA SILVA e LEÃO (1979), a EUEM é variável para os diferentes processos fisiológicos, devido ao fato de as exigências energéticas serem influenciadas pelo clima, trabalho muscular e, principalmente, pela concentração de EM da dieta.

As variações no balanço da energia são dependentes do nível de ingestão de alimentos, que é influenciado por interações entre os alimentos, denominadas efeito associativo. A adição de concentrado a dietas volumosas aumenta parcialmente a EUEM para mantença e ganho (NRC, 1984). Em virtude da depressão na produção de metano, redução da ruminação e diminuição do incremento calórico (VAN SOEST, 1994), a eficiência de utilização da energia ingerida tende a ser maior para dietas concentradas, quando comparadas a volumosas, devido aos menores requerimentos líquidos de mantença (AGRICULTURAL RESEARCH COUNCIL ARC, 1980). Também, alimentos volumosos de melhor qualidade são mais eficientes que os de pior qualidade (VAN SOEST, 1994).

Nos ruminantes, os produtos finais da fermentação fornecem a maior fonte de energia, porém, quando carboidratos são fermentados no rúmen, ocorrem perdas de energia na forma de calor de fermentação e metano. A EUEM para ganho é negativamente correlacionada com o conteúdo de fibra na dieta e tem sido especulado que este comportamento seja devido à associação do conteúdo de fibra da dieta com o tipo de fermentação no rúmen e os produtos finais formados, mais que propriamente devido ao trabalho físico exercido na ruminação e digestão do material fibroso (COELHO DA SILVA e LEÃO, 1979; BLACK et al., 1987). Contudo, segundo GARRETT (1980a), os processos de ingestão e digestão de alimentos são responsáveis por cerca de 25 a $30 \%$ do total do incremento calórico.

A eficiência para síntese de gordura decresce com o aumento da proporção de ácido acético (COELHO DA SILVA e LEÃO, 1979), pois a síntese de ácidos graxos a partir do acetato requer $\mathrm{NADPH}_{2} \mathrm{e}$, segundo Armstrong (1965), citado por BLACK et al. (1987), inadequado suprimento de $\mathrm{NADPH}_{2}$ pode limitar a conversão de acetato para ácidos graxos em animais alimentados com dietas ricas em volumosos.

Estes equivalentes reduzidos são produzidos mais eficientemente via ciclo das pentoses fosfatadas que pela isocitrato desidrogenase (BALDWIN et al., 1976), face à maior disponibilidade de substrato glicogênico (OLDHAM, 1996). Quando o suprimento de $\mathrm{NADPH}_{2}$ é inadequado, energia é perdida como calor em algumas formas de ciclos fúteis e a eficiência da utilização da energia, reduzida (BLACK et al., 1987). Então, quando a proporção de propionato é aumentada, maior é a disponibilidade de glicose para gerar equivalentes reduzidos.

Diferenças nas taxas metabólicas dos vários órgãos e vísceras podem também influenciar a utilização da energia. CATON e DHUYVETTER (1997) relataram que, embora os tecidos viscerais compreendam pequena porção do peso corporal, eles consomem, aproximadamente, $50 \%$ da energia para mantença. Por outro lado, o tecido muscular, que constitui cerca de $41 \%$ da massa corporal, consome apenas $23 \%$ do total da energia requerida para mantença.

O NRC (1984) apresentou valores de EUEM para mantença entre 57,6 e 68,6\% e para ganho de 29 a $47,3 \%$, para rações com diferentes proporções volumoso:concentrado, cujos teores de EM variaram de 2,0 a 3,2 Mcal $/ \mathrm{kg}$ MS.

O AGRICULTURAL RESEARCH COUNCIL ARC (1980) desenvolveu equações lineares para o cálculo das EUEM, para mantença e crescimento, a partir da metabolizabilidade da energia bruta da dieta, a qual não é constante, mas diminui conforme o nível de alimentação aumenta. Este sistema e o AGRICULTURAL AND FOOD RESEARCH COUNCIL - AFRC (1993) consideram redução na EUEM para retenção de energia, com o aumento da ingestão. 
906 Rev. bras. zootec.

O decréscimo da metabolizabilidade da dieta com o aumento do nível de ingestão pode ser atribuído ao aumento na taxa de passagem, diminuindo a digestão do amido e de carboidratos da parede celular, com conseqüente diminuição da digestibilidade e aumento das perdas fecais e, ou, aumento nos requisitos de mantença, quando o nível de consumo é alto, além de diferenças na eficiência de síntese de proteína e gordura (GEAY, 1984).

As diferenças na EUEM para ganho dependem das variações na composição do ganho (GARRETT, 1980a; OLDHAM, 1996). GARRETT (1980a) relatou que a EUEM para síntese de proteína oscila entre 10 e $40 \%$, enquanto para síntese de gordura, entre 60 e $80 \%$. GEAY (1984) citou eficiência de síntese de proteína e gordura de 20 e $75 \%$, respectivamente. Por outro lado, OWENS et al. (1995) relataram eficiência de acréscimo de proteína de $47 \%$ e, para síntese de gordura, de $76 \%$.

Estes valores indicam que a deposição de proteína é energeticamente menos eficiente que a síntese de gordura, o que se deve ao fato de o processo de síntese e degradação (turnover) da proteína corporal reduzir a eficiência energética de seu acúmulo (GARRETT, 1980a; GEAY,1984; OWENS et al., 1995; e FONTES, 1995).

No Brasil, embora já tenham sido conduzidos vários trabalhos para a determinação das exigências nutricionais de bovinos de corte, poucos se referem às exigências dietéticas destes animais, e a expressão mais comumente utilizada para determinar o valor energético dos alimentos é na forma de nutrientes digestíveis totais. Portanto, o objetivo do presente trabalho foi determinar as EUEM e as exigências de energia metabolizável e de nutrientes digestíveis totais, para mantença e ganho de peso, de bovinos Nelore, submetidos a cinco tratamentos com diferentes níveis de concentrado na dieta.

\section{Material e Métodos}

O local do experimento, as instalações, as dietas (proporção dos ingredientes e composição), o sistema de alimentação, o manejo dos animais, os procedimentos de abate, as coletas e as análises químicas dos alimentos e dos tecidos, o delineamento experimental e as análises estatísticas foram descritos por VÉRAS et al. (2000a,b).

Foram utilizados 35 bovinos Nelore, não-castrados, com idade média de 20 meses e peso vivo médio inicial de $330 \mathrm{~kg}$.
As concentrações de energia líquida das dietas foram calculadas segundo HARRIS (1970).

Os valores de EM da dieta foram calculados considerando-se que $1 \mathrm{~kg}$ de nutrientes digestíveis totais (NDT) é igual a 4,409 Mcal de energia digestível (ED) e 1 Mcal de ED, a 0,82 Mcal de EM (COELHO DA SILVA e LEÃO, 1979). Os teores de NDT foram obtidos por VÉRAS et al. (1999a), segundo SNIFFEN et al. (1992).

O consumo de matéria seca (MS) suficiente para manter o equilíbrio de energia foi calculado dividindo-se o consumo de EM suficiente para mantença, de 147 kcal/kg0,75 (VÉRAS et al., 1999b), pela concentração de EM (kcal/kg de MS) da dieta, em cada tratamento (VÉRAS et al., 1999a).

A concentração de energia líquida de cada ração para mantença (ELm) foi obtida dividindo-se a produção de calor em jejum, de $82,79 \mathrm{kcal} / \mathrm{kgPCVZ}^{0,75}$, pelo consumo de MS, para manter o equilíbrio de energia, expresso em $\mathrm{g}$ de $\mathrm{MS} / \mathrm{kg}^{0,75}$, enquanto o consumo de MS acima das necessidades de mantença foi obtido subtraindo-se do consumo total de MS (gMS/ $/ \mathrm{kg}^{0,75}$ ) o consumo de MS suficiente para o equilíbrio de energia $\left(\mathrm{gMS} / \mathrm{kg}^{0,75}\right)$, para cada ração, e a concentração de ELg foi calculada dividindo-se a energia retida por dia, em $\mathrm{kcal} / \mathrm{kg}^{0,75}$, pelo consumo de MS acima das necessidades de mantença, expresso em $\mathrm{gMS} / \mathrm{kg}^{0,75}$. Os dados foram obtidos por VÉRAS et al. (1999b).

As EUEM para mantença $(\mathrm{km})$ e ganho de peso (kf) foram estimadas a partir da regressão entre os teores de energia líquida, para mantença ou ganho, respectivamente, em função da EM da dieta, segundo GARRETT (1980b), sendo que a kf também foi estimada como o coeficiente de regressão linear entre a ER e o CEM, para os animais de cada tratamento, segundo o NRC (1996) e FERRELL e JENKINS (1998a,b), juntamente com os dados relativos aos dos animais designados de mantença.

Para a estimativa da kf, segundo GARRETT (1980b), foram utilizados os dados obtidos no presente estudo e, para conferir maior robustez, foi efetuada outra equação, na qual foram usados somente os dados que demonstraram aumento da ELg com o acréscimo dos teores de EM das dietas, ou seja, foram utilizados os seguintes valores: 0,$53 ; 0,83 ; 0,9$; 1,09 ; e $1,27 \mathrm{Mcal} / \mathrm{kg}$ de MS de ELg, relativos às concentrações de EM de 2,28; 2,35; 2,43; 2,51; e $2,59 \mathrm{Mcal} / \mathrm{kg}$ de MS, respectivamente, sendo o primeiro e terceiro valores obtidos por FERREIRA et al. (1998), que utilizaram ingredientes e proporções de 
concentrado semelhantes às do presente trabalho, para bovinos F1 Simental x Nelore, não-castrados.

Os requisitos de EM para mantença e ganho foram obtidos pelas relações entre as exigências líquidas e as respectivas EUEM estimadas segundo GARRETT (1980b), para os dados conjuntos do presente trabalho e os de FERREIRA et al. (1998). As exigências de NDT foram calculadas dividindo-se as exigências de EM por 0,82, obtendo-se as exigências de energia digestível (ED) e, posteriormente, dividindo-se as exigências de ED por 4,409.

Para predição do PCVZ a partir do PV, foi utilizada a equação geral obtida por VÉRAS et al. (1999b), ajustada para todos os dados: $\mathrm{PCVZ}=-46,262+0,9911 \mathrm{PV} ; \mathrm{r}_{2}=0,94$. O fator para conversão das exigências para ganho de PCVZ em exigências para ganho de PV obtido a partir desta equação foi igual a 1 .

\section{Resultados e Discussão}

Na Tabela 1, são apresentados os teores de NDT, as concentrações de EM das dietas e os valores calculados de ELm, ELg, além das km e kf calculadas e as estimativas das kf, segundo o NRC (1996), FERRELL e JENKINS (1998a,b) e GARRETT (1980a,b).

O NRC (1984) estimou as concentrações de ELm e de ELg de uma ração com teor de EM de 2,60 Mcal/kg MS, em 1,69 e 1,08 Mcal/kg MS, respectivamente, e as $\mathrm{km}$ e $\mathrm{kf}$ estimadas para uma dieta com esta mesma concentração de EM, segundo este conselho, seriam de 65,1 e 41,5\%. Estes valores diferem dos obtidos no presente trabalho, para uma dieta com teor de EM de 2,59 Mcal/kg MS, que foram 1,46 e 1,27 Mcal/kg MS, para ELm e ELg, e 56 e $49 \%$, para $\mathrm{km}$ e $\mathrm{kf}$, respectivamente.

As equações que geraram os resultados para a estimativa das $\mathrm{kf}$ como sendo os coeficientes de regressão das relações lineares entre a ER (Y) e o CEM (X) para os animais de cada tratamento, segundo preconizado pelo NRC (1996) e FERRELL e JENKINS (1998a,b), foram: $Y=-42,693+$ $0,3227 \mathrm{X}, \mathrm{r}^{2}=0,44 ; \mathrm{Y}=-45,517+0,3266 \mathrm{X}$, $\mathrm{r}^{2}=0,79 ; \quad \mathrm{Y}=-64,85+0,438 \mathrm{X}, \mathrm{r}^{2}=0,89$; $\mathrm{Y}=-73,736+0,4939 \mathrm{X}, \mathrm{r}^{2}=0,86$ e $\mathrm{Y}=-107,34$ $+0,6924 \mathrm{X}, \mathrm{r} 2=0,95$. Portanto, as kf estimadas, segundo os referidos autores, foram iguais a 0,32 ; 0,$33 ; 0,44 ; 0,49$; e 0,69 , respectivamente, para as concentrações de EM de 2,$11 ; 2,35 ; 2,51 ; 2,59$; e 2,52 Mcal/kg de MS.

A equação ajustada relacionando as concentrações de Elg, em função das concentrações de EM das dietas do presente trabalho, segundo GARRETT (1980b), foi:

$$
\mathrm{Y}=-54,764 \mathrm{EM}^{3}+391,28 \mathrm{EM}^{2}-927,84 \mathrm{EM}+
$$
$731,13, \mathrm{R}^{2}=0,64$. Os valores estimados para $\mathrm{kf}$ foram determinados a partir da relação entre a ELg estimada pela referida equação e as concentrações de EM das dietas, cujos percentuais foram $45 ; 35 ; 54 ; 50$; e 54\%, respectivamente, para as concentrações de EM de 2,$11 ; 2,35 ; 2,51 ; 2,59$; e 2,52 Mcal/kg de MS das dietas, respectivamente.

Tabela 1 - Teores de nutrientes digestíveis totais (NDT), concentrações de energia metabolizável (EM), energia líquida para mantença (ELm), energia líquida para ganho (ELg) e eficiências de utilização da energia metabolizável (EUEM) calculadas para mantença $(\mathrm{km})$ e EUEM calculadas e estimadas para ganho de peso (kf), em função do nível de concentrado (NC) na dieta

Table 1 - Contents of total digestible nutrients (TDN), metabolizable energy (ME) concentrations, net energy maintenance (NEm), net energy for gain (NEg) and efficiencies of metabolizable energy utilization (EMEU) calculated and estimated for weight gain (kf), in function of the level of concentrate in diet

\begin{tabular}{|c|c|c|c|c|c|c|c|c|}
\hline \multirow[t]{2}{*}{$\begin{array}{c}\mathrm{NC}(\%) \\
L C\end{array}$} & \multirow[t]{2}{*}{$\begin{array}{c}\operatorname{NDT}(\%) \\
T D N\end{array}$} & \multicolumn{3}{|c|}{$\begin{array}{c}\mathrm{Mcal} / \mathrm{kg} \text { de } \mathrm{MS} \\
\text { Mcal/kg DM }\end{array}$} & \multicolumn{4}{|c|}{$\begin{array}{c}\operatorname{EUEM}(\%) \\
E M E U\end{array}$} \\
\hline & & EM & ELm & ELg & $\mathrm{km}^{1}$ & $\mathrm{kf}^{1}$ & $\mathrm{kf}^{2}$ & $\mathrm{kf}^{3}$ \\
\hline 25 & 58,42 & 2,11 & 1,19 & 0,95 & 56 & 45 & 32 & 4 \\
\hline 37,5 & 65,08 & 2,35 & 1,32 & 0,83 & 56 & 35 & 33 & 35 \\
\hline 50 & 69,69 & 2,51 & 1,42 & 1,09 & 56 & 43 & 44 & 54 \\
\hline 62,5 & 71,80 & 2,59 & 1,46 & 1,27 & 56 & 49 & 49 & 50 \\
\hline 75 & 69,62 & 2,52 & 1,41 & 1,63 & 56 & 65 & 69 & 54 \\
\hline
\end{tabular}

\footnotetext{
1 Calculadas a partir dos dados do presente trabalho.

2 Estimadas segundo o NRC (1996) e FERRELL e JENKINS (1998a,b) utilizando-se dados do presente trabalho.

3 Estimadas segundo GARRETT (1980a,b) utilizando-se dados do presente trabalho.

1 Calculated from the data of the present work.

2 Estimated according to NRC (1996) and FERRELL and JENKINS (1998a,b) using data from the present work.

3 Estimated according to GARRETT (1980a,b) using data from the present work.
} 
908 Rev. bras. zootec.

FERRELL e JENKINS (1998a,b), a partir de relação não-linear entre a ER e o CEM, concluíram que a kf não foi constante, mas decresceu à medida que o CEM aumentou. A equação obtida para esta relação por VÉRAS et al. (1999b) indicou que a energia no ganho aumentou de forma não-linear, conforme a crescente ingestão de EM, e alcançaria o máximo de, aproximadamente, $90 \mathrm{kcal} / \mathrm{kg}^{0,75} / \mathrm{dia}$, permitindo também inferir que a kf é variável e dependente da ingestão de EM. O máximo consumo de energia, expresso em $\mathrm{kg} / \mathrm{dia}$ de NDT, obtido por VÉRAS et al. (1999a), foi 6,07 kg/dia, estimado com $59,45 \%$ de concentrado na dieta, o que corresponde ao consumo de 21,95 Mcal/dia de EM.

A equação ajustada para estimativa da kf, segundo GARRETT (1980b), relacionando as concentrações de ELg (Y), em função das concentrações de EM (X) das dietas obtidas no presente estudo $(2,35 ; 2,51$; e $2,59 \mathrm{Mcal} / \mathrm{kg}$ de MS) e por FERREIRA et al. (1998), de 2,28 e 2,43 Mcal/kg de MS, foi: $\mathrm{Y}=43,099 \mathrm{EM} 3-316,65 \mathrm{EM} 2+776,79$ $\mathrm{EM}-635,31, \mathrm{R}^{2}=0,99$. As kf estimadas a partir da substituição dos valores de EM das dietas deste experimento na referida equação foram, respectivamente, $23 ; 33 ; 38 ; 41 ;$ e $49 \%$. A relação entre a ELg estimada e a EM das dietas é apresentada na Figura 1.

As exigências de EM, ED e NDT para mantença, para diferentes pesos vivos e de corpo vazio, são demonstradas na Tabela 2, na qual se observam

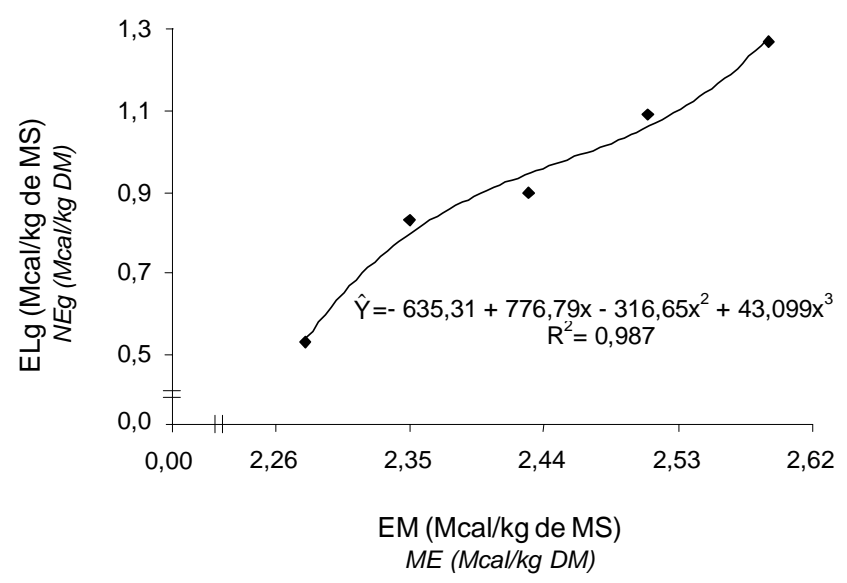

Figura 1 - Estimativa da energia líquida de ganho (ELg), expressa em Mcal/kg MS, em função da concentração de energia metabolizável (EM) das dietas, expressa em Mcal/kgMS.

Figure 1 - Estimate of net energy for gain (NEg), express in Mcal/ $\mathrm{kg} D M$, in function of the level of metabolizable energy (ME) in the diets, express in Mcal/kg DM. aumentos nestes requisitos, à medida que o peso corporal se elevou, apresentando a mesma tendência das exigências líquidas de energia para mantença relatadas por VÉRAS et al. (1999b).

Considerando-se a $\mathrm{km}$ obtida de 0,56 e o valor de $82,79 \mathrm{kcal} / \mathrm{kgPCVZ}^{0,75}$, como requisito líquido de energia para mantença (VÉRAS et al., 1999b), os requerimentos de EM seriam 147,84 kcal/ $\mathrm{PCVZ}^{0,75}$; os de ED, 180,29 kcal/PCVZ ${ }^{0,75}$; e os de NDT, 40,89 g/PCVZ ${ }^{0,75}$.

Na Tabela 3, são apresentadas as estimativas dos requerimentos de EM, para ganho de peso, em Mcal por $\mathrm{kg}$ de ganho de peso do corpo vazio (Mcal/kg GPCVZ), e de NDT, em kg/kg de GPCVZ, utilizando-se a relação entre os requisitos líquidos estimados para os dados em conjunto por VÉRAS et al. (1999b) e as kf para as concentrações de EM de 2,4 e 2,6 $\mathrm{Mcal} / \mathrm{kg}$ de MS, estimadas a partir da equação conjunta no presente trabalho. Apenas foram utilizados os valores relativos às exigências líquidas de energia dos dados em conjunto, porque o teste de identidade de modelos efetuado por VÉRAS et al. (1999b) indicou não haver diferença entre os tratamentos.

As exigências de EM aumentaram, à medida que o peso corporal se elevou, o que concorda com o AFRC (1993), que estimou aumento nos requerimentos de EM para ganho de $1 \mathrm{~kg}$, de 79 para $95 \mathrm{MJ} / \mathrm{dia}$, com a elevação do peso corporal de 300 para $400 \mathrm{~kg}$, respectivamente.

Tabela 2 - Requisitos diários de energia metabolizável (EM) e energia digestível (ED), expressos em Mcal/ dia, e requisitos de NDT ( $\mathrm{kg} / \mathrm{dia})$, para mantença de bovinos Nelore, em função do peso vivo (PV) ou do peso do corpo vazio (PCVZ)

Table 2 - Average requirements of metabolizable energy (ME) and digestible energy (DE), express in Mcal/day, and TDN requirements, for maintenance of Nellore bulls, in function of live weight (LW) or empty body weight (EBW)

\begin{tabular}{|c|c|c|c|c|}
\hline $\begin{array}{l}\mathrm{PV}(\mathrm{kg}) \\
L W\end{array}$ & $\begin{array}{c}\text { PCVZ } \\
(\mathrm{kg}) \\
E B W\end{array}$ & $\begin{array}{c}\text { EM } \\
\text { (Mcal/dia) } \\
M E \\
\text { (Mcal/day) }\end{array}$ & $\begin{array}{c}\mathrm{ED} \\
\text { (Mcal/dia) } \\
D E \\
\text { (Mcal/day) }\end{array}$ & $\begin{array}{c}\text { NDT } \\
\text { (kg/dia) } \\
T D N \\
\text { (kg/day) }\end{array}$ \\
\hline 300 & 251,07 & 9,32 & 11,37 & 2,58 \\
\hline 350 & 300,62 & 10,68 & 13,02 & 2,95 \\
\hline 400 & 350,18 & 11,94 & 14,56 & 3,30 \\
\hline 450 & 399,73 & 13,21 & 16,11 & 3,65 \\
\hline
\end{tabular}


VÉRAS et al.

Tabela 3 - Estimativa dos requisitos de energia líquida para ganho de peso (ELg), em Mcal/kg de ganho de peso de corpo vazio (GPCVZ), de energia metabolizável (EM), em Mcal/kg GPCVZ, e de nutrientes digestíveis totais (NDT), em kg/kg GPCVZ, em função das concentrações de EM da dieta (Mcal/kg de MS), e respectivas eficiências de utilização da energia metabolizável para ganho de peso (kf), em \%, de bovinos Nelore para diferentes pesos vivo (PV) e de corpo vazio (PCVZ)

Table 3 - Estimate of the net energy requirement for weight gain (NEg), in Mcal/kg of empty body weight gain (EBWG), of metabolizable energy (ME), in Mcal/kg EBWG, and of total digestible nutrients (TDN), in $\mathrm{kg} / \mathrm{kg}$ EBWG, and respective efficiencies of metabolizable energy utilization for weight gain (kf), in \%, of Nellore bulls, for the different live weight (LW) and the empty body weight (EBW)

\begin{tabular}{|c|c|c|c|c|c|c|c|}
\hline \multirow{4}{*}{$\begin{array}{l}\mathrm{PV}(\mathrm{kg}) \\
L W\end{array}$} & \multirow{4}{*}{$\begin{array}{c}\mathrm{PCVZ}(\mathrm{kg}) \\
E B W\end{array}$} & \multicolumn{6}{|c|}{$\begin{array}{l}\text { EM da dieta } \\
M E \text { of the diet }\end{array}$} \\
\hline & & \multicolumn{2}{|c|}{$2,4(\mathrm{kf}=0,37)$} & & \multicolumn{3}{|c|}{$2,6(\mathrm{kf}=0,50)$} \\
\hline & & \multicolumn{6}{|c|}{$\begin{array}{c}\text { Exigência } \\
\text { Requirement }\end{array}$} \\
\hline & & $\mathrm{ELg}^{1}$ & EM & NDT & ELg & EM & NDT \\
\hline 300 & 251,07 & 3,26 & 8,81 & 2,44 & 3,26 & 6,52 & 1,80 \\
\hline 350 & 300,62 & 3,46 & 9,35 & 2,59 & 3,46 & 6,92 & 1,91 \\
\hline 400 & 350,18 & 3,64 & 9,84 & 2,72 & 3,64 & 7,28 & 2,01 \\
\hline 450 & 399,73 & 3,80 & 10,27 & 2,84 & 3,80 & 7,60 & 2,10 \\
\hline
\end{tabular}

1 VÉRAS et al. (2000b)

As exigências totais de EM (mantença + ganho de $1 \mathrm{~kg}$ de PCVZ) de um animal pesando $400 \mathrm{~kg}$, alimentado com dietas com teor de EM de 2,4 Mcal/kgMS, foram 21,78 e, para dietas com concentração de EM de 2,6 Mcal/kgMS, 19,22 Mcal/dia. Também, os requisitos de NDT para um animal com o mesmo PV foram 6,02 e $5,31 \mathrm{~kg}$, para dietas com concentrações de EM de 2,4 e 2,6 Mcal/kgMS, respectivamente, permitindo inferir que, quanto maior a EUEM para ganho de peso, menores os requisitos de EM e de NDT.

Os requerimentos totais de NDT (mantença + ganho de peso) foram próximos ao recomendado pelo NRC (1984), para um bovino não-castrado de porte médio, com peso aproximado de $400 \mathrm{~kg}$ (900 lb), ganhando, aproximadamente, $1 \mathrm{~kg} / \mathrm{dia}(2 \mathrm{lb})$, que é cerca de $6,0 \mathrm{~kg} / \mathrm{dia}(13,23 \mathrm{lb})$.

\section{Conclusões}

A eficiência de utilização da energia metabolizável para mantença observada foi de $56 \%$.

Recomenda-se o uso da equação: $\mathrm{ELg}=43,099$ $\mathrm{EM}^{3}-316,65 \mathrm{EM}^{2}+776,79 \mathrm{EM}-635,31$, para estimativa da eficiência de utilização da energia metabolizável para ganho de peso de zebuínos e F1 taurinos x zebuínos, não-castrados.

\section{Referências Bibliográficas}

AGRICULTURAL AND FOOD RESEARCH COUNCIL AFRC. 1993. Energy and protein requeriments of ruminants. Wallingford: Commonwealth Agricultural Bureaux International. $159 \mathrm{p}$.

AGRICULTURAL RESEARCH COUNCIL - ARC. 1980. The nutrient requirements of ruminants livestock. London: Commonwealth Agricultural Bureaux. 351p.

BALDWIN, R.L., YANG, K.C., GRICHTING, G. 1976. Theoretical model of ruminant adipose tissue in relation to the whole animal. Fed. Proc., 35(11):2314-2318.

BLACK, J.L., GILL, M., BEEVER, D.E. et al. 1987. Simulation of the metabolism of absorved energy-yielding nutrients in young sheep: Efficiency of utilization of acetate. J. Nutr., 117:105-115.

CATTON, J.S., DHUYVETTER, D.V. 1997. Influence of energy supplementation on grazing ruminants: Requirements and responses. J. Anim. Sci., 75:533-542.

COELHO DA SILVA, J.F., LEÃO, M.I. 1979. Fundamentos de nutrição dos ruminantes. Piracicaba: Livroceres. 380p.

FERREIRA, M.A., VALADARES FILHO, S.C., VALADARES, R.F.D. et al. Eficiência de utilização da energia metabolizável e exigências de energia metabolizável e NDT de bovinos de corte. In: REUNIÃO ANUAL DA SOCIEDADE BRASILEIRA DE ZOOTECNIA, 35, 1998, Botucatu, SP. Anais... Botucatu: SBZ, 1998. p.269-271.

FERRELL, C.L., JENKINS, T.G. 1998a. Body composition and energy utilization by steers of diverse genotypes fed a highconcentrate diet during the finishing period: I. Angus, Belgian Blue, Hereford, and Piedmontese Sires. J. Anim. Sci., 76:637-646.

FERRELL, C.L., JENKINS, T.G. 1998b. Body composition and energy utilization by steers of diverse genotypes fed a high- 
910 Rev. bras. zootec.

concentrate diet during the finishing period: I. Angus, Belgian Blue, Hereford, and Piedmontese Sires. J. Anim. Sci., 76:647-657.

FONTES, C.A.A. Composição corporal, exigências líquidas de nutrientes para ganho de peso e desempenho produtivo de animais zebuínos e mestiços europeu-zebu. Resultados experimentais. In: PEREIRA, J.C. (Ed.). SIMPÓSIO INTERNACIONAL SOBRE EXIGÊNCIAS NUTRICIONAIS DE RUMINANTES, 1995, Viçosa, MG. Anais... Viçosa, MG: DZO, 1995. p.419-455.

GARRETT, W.N. Energy utilization by growing cattle as determined in 72 comparative slaughter experiments. 1980. In: SYMPOSIUM OF ENERGY METABOLISM, 8, Cambridge, 1980. Proceedings... Butterworths, London: EAAP Publ. n.28. 1980a. p.3-7.

GARRETT, W.N. 1980b. Factors influencing energetic efficiency of beef production. J. Anim. Sci., 51(6):1434-1440.

GEAY, Y. 1984. Energy and protein utilization in growing cattle. J. Anim. Sci., 58(3):766-778.

HARRIS, L.F. 1970. Nutrition research technique for domestic and wild animal. v.1, Logan, Utah, paginação descontínua.

NATIONAL RESEARCH COUNCIL - NRC. 1984. Nutrient requeriments of beef cattle. 6 . ed. Washington, D.C. 90p.

NATIONAL RESEARCH COUNCIL - NRC. 1996. Nutrient requirements of beef cattle. 7.ed. Washington, D.C. 242p.

OLDHAM, J.D. 1996. Nutrient allowances for ruminants. In: GARNSWORTHY, P.C., COLE, D.J.A. (Eds.). Recent developments in ruminant nutrition. UK: Nottingham University. p.1-22.
OWENS, F.N., GILL, D.R., SECRIST, D.S. et al. 1995. Review of some aspects of growth and devepopment of feedlot cattle. J. Anim. Sci., 73:3152-3172.

SNIFFEN, C.J., O'CONNOR, J.D., VAN SOEST, P.J. et al. 1992. A net carbohydrate and protein system for evaluating cattle diets: II. Carbohydrate and protein availability. J. Anim. Sci., 70:3562-3577.

VAN SOEST, P.J. 1994. Nutritional ecology of the ruminant. 2.ed. Ithaca: Cornell University Press. 476p.

VÉRAS, A.S.C., VALADARES FILHO, S.C.V., COELHO DA SILVA, J.F. et al. 2000a. Consumo e digestibilidade aparente em bovinos Nelore não-castrados, alimentados com diferentes níveis de concentrado na dieta. Rev. bras. zootec., 29(6)2367-2378 (supl. 2).

VÉRAS, A.S.C., VALADARES FILHO, S.C.V, COELHO DA SILVA, J.F. et al. 2000b. Composição corporal e requisitos energéticos e protéicos de bovinos Nelore não-castrados alimentados com diferentes níveis de concentrado na dieta. Rev. bras. zootec., 29(6):2379-2389 (supl. 2).

Recebido em: 26/11/99

Aceito em: 11/12/00 